 \\ TATRA \\ MOUNTaiNS \\ Mathematical Publications
}

\section{FUNCTIONAL EQUATIONS STEMMING FROM PROBABILITY THEORY}

\author{
KÁroly LaJkó - FruZsina MÉszÁros
}

ABSTRACT. Special cases of the functional equation

$$
h_{1}\left(\frac{x}{c(y)}\right) \frac{1}{c(y)} f_{Y}(y)=h_{2}\left(\frac{y}{d(x)}\right) \frac{1}{d(x)} f_{X}(x)
$$

are investigated for almost all $(x, y) \in \mathbb{R}_{+}^{2}$, for the given functions $c, d$ and the unknown functions $h_{1}, h_{2}, f_{X}$ and $f_{Y}$.

\section{Introduction}

Functional equations have many interesting applications in characterization problems of probability theory.

In 11 Arnold, Castillo and Sarabia showed how solutions of functional equations can be used in characterizing joint distributions from conditional distributions. They considered, among others, all possible distributions with given regression functions with conditionals in scale families.

They obtained those equations in the following way.

Let $(X, Y)$ be an absolutely continuous bivariate random variable whose joint, marginal and conditional density functions are denoted by $f_{(X, Y)}$ and $f_{X}, f_{Y}$, $f_{X \mid Y}, f_{Y \mid X}$, respectively. One can write $f_{(X, Y)}$ in two different ways and obtain the functional equation

$$
f_{(X, Y)}(x, y)=f_{X \mid Y}(x, y) f_{Y}(y)=f_{Y \mid X}(x, y) f_{X}(x)
$$

for all $(x, y) \in \mathbb{R}^{2}$ (or for all $(x, y) \in \mathbb{R}_{+}^{2}$ if we restrict our investigations to the random variable $(X, Y)$ with support in the positive quadrant).

They studied joint densities whose conditional densities satisfy

$$
f_{X \mid Y}(x, y)=h_{1}\left(\frac{x}{c(y)}\right) \frac{1}{c(y)}
$$

2000 Mathematics Subject Classification: 39B22, 62 E10.

Keywords: characterizations of probability distributions, measurable solution a.e.

Research supported by OTKA, Grant No. NK 68040. 


\section{KÁROLY LAJKÓ — FRUZSINA MÉSZÁROS}

and

$$
f_{Y \mid X}(x, y)=h_{2}\left(\frac{y}{d(x)}\right) \frac{1}{d(x)}
$$

for given positive functions $c$ and $d$, where $h_{1}, h_{2}$ are positive unknown functions.

Then from (1) one can deduce the functional equation

$$
h_{1}\left(\frac{x}{c(y)}\right) \frac{1}{c(y)} f_{Y}(y)=h_{2}\left(\frac{y}{d(x)}\right) \frac{1}{d(x)} f_{X}(x) .
$$

For a special choice of the given functions, A r nold, C a stillo and S a r a bi a solved (4) assuming the existence of derivatives of the unknown functions $h_{1}, h_{2}$, $f_{Y}, f_{X}$ up to the second order. They restricted the search to a random variable $(X, Y)$ with support in the positive quadrant and thereby it was possible to determine the nature of the joint distribution.

In this paper, under special choices of the given functions, we assume only the measurability of the positive unknown functions $h_{1}, h_{2}, f_{Y}, f_{X}$ and that the so obtained equations hold for almost all pairs $(x, y)$ from $\mathbb{R}_{+}^{2}$.

We prove that the measurable solutions of (44) satisfied almost everywhere - in the different special cases - can uniquely be extended to continuous functions and when the measurable functions are replaced with the continuous functions, equation (4) is satisfied everywhere on $\mathbb{R}_{+}^{2}$.

Here, we will use the following result of A. Jár a i (see [3] and [4]).

Theorem 1 ( $\mathrm{J}$ ár a i). Let $Z$ be a regular topological space, $Z_{i}(i=1,2, \ldots, n)$ be topological spaces and $T$ be a first countable topological space. Let $Y$ be an open subset of $\mathbb{R}^{k}, X_{i}$ an open subset of $\mathbb{R}^{r_{i}}, r_{i} \in \mathbb{Z},(i=1,2, \ldots, n)$ and $D$ an open subset of $T \times Y$. Let furthermore $T^{\prime} \subset T$ be a dense subset, $f: T^{\prime} \rightarrow Z$, $g_{i}: D \rightarrow X_{i}$ and $h: D \times Z_{1} \times \ldots \times Z_{n} \rightarrow Z$. Suppose that the function $f_{i}$ is almost everywhere defined on $X_{i}$ (with respect to the $r_{i}$-dimensional Lebesgue measure) with values in $Z_{i}(i=1,2, \ldots n)$ and the following conditions are satisfied:

(1) for all $t \in T^{\prime}$ and for almost all $y \in D_{t}=\{y \in Y \mid(t, y) \in D\}$

$$
f(t)=h\left(t, y, f_{1}\left(g_{1}(t, y)\right), \ldots, f_{n}\left(g_{n}(t, y)\right)\right) ;
$$

(2) for each fixed $y$ in $Y$, the function $h$ is continuous in the other variables;

(3) $f_{i}$ is Lebesgue measurable on $x_{i}(i=1,2, \ldots, n)$;

(4) $g_{i}$ and the partial derivative $\frac{\partial g_{i}}{\partial y}$ are continuous on $D(i=1,2, \ldots, n)$;

(5) for each $t \in T$ there exist a $y$ such that $(t, y) \in D$ and the partial derivative $\frac{\partial g_{i}}{\partial y}$ has the rank $r_{i}$ at $(t, y) \in D(i=1,2, \ldots, n)$.

Then there exists a unique continuous function $\tilde{f}$ such that $f=\tilde{f}$ almost everywhere on $T$, and if $f$ is replaced with $\tilde{f}$ then equation (5) is satisfied almost everywhere on $D$. 


\section{FUNCTIONAL EQUATIONS STEMMING FROM PROBABILITY THEORY}

\section{First problem}

Let us consider the case when the functions $c, d$ are of the form

$$
c(y)=\frac{1}{\alpha+y}, \quad d(x)=\frac{1}{\beta+x} \quad(x, y>0),
$$

where $\alpha, \beta$ are non-negative constants.

From (4) we get the equation

$$
h_{1}((\alpha+y) x)(\alpha+y) f_{Y}(y)=h_{2}((\beta+x) y)(\beta+x) f_{X}(x)
$$

for almost all $(x, y) \in \mathbb{R}_{+}^{2}$, where $h_{1}, h_{2}, f_{X}, f_{Y}: \mathbb{R}_{+} \rightarrow \mathbb{R}_{+}$are measurable unknown functions, $\alpha, \beta \geq 0$ are arbitrary constants.

Easy calculation shows the validity of the following technical lemma.

LEMMA 1. The positive measurable functions $h_{1}, h_{2}, f_{X}, f_{Y}$ satisfy equation (6) for almost all $(x, y) \in \mathbb{R}_{+}^{2}$ if and only if the measurable functions $G_{1}, G_{2}, F_{1}$, $F_{2}: \mathbb{R}_{+} \rightarrow \mathbb{R}$ defined by

$$
\begin{array}{ll}
G_{1}(t)=\ln \left[h_{1}(t)\right], & G_{2}(t)=\ln \left[h_{2}(t)\right], \\
F_{1}(t)=\ln \left[(\alpha+t) f_{Y}(t)\right], & F_{2}(t)=\ln \left[(\beta+t) f_{X}(t)\right], \quad\left(t \in \mathbb{R}_{+}\right)
\end{array}
$$

satisfy the functional equation

$$
G_{1}(x(\alpha+y))+F_{1}(y)=G_{2}(y(\beta+x))+F_{2}(x),
$$

for almost all $(x, y) \in \mathbb{R}_{+}^{2}$, where $\alpha, \beta \geq 0$ are arbitrary constants.

To get the measurable solution of equation (7) (and so (6) ) satisfied almost everywhere, we distinguish 2 cases:

(1) $\alpha^{2}+\beta^{2} \neq 0$;

(2) $\alpha=\beta=0$.

2.1. The $\alpha^{2}+\beta^{2} \neq 0$ case

In this case, with help of Theorem 1, we can prove the following

TheOREM 2. If the measurable functions $G_{1}, G_{2}, F_{1}, F_{2}: \mathbb{R}_{+} \rightarrow \mathbb{R}$ satisfy equation (77) for almost all $(x, y) \in \mathbb{R}_{+}^{2}$, then there exist unique continuous functions $\widetilde{G}_{1}, \widetilde{G}_{2}, \widetilde{F}_{1}, \widetilde{F}_{2}: \mathbb{R}_{+} \rightarrow \mathbb{R}$ such that $\widetilde{G}_{1}=G_{1}, \widetilde{G}_{2}=G_{2}, \widetilde{F}_{1}=F_{1}$ and $\widetilde{F}_{2}=F_{2}$ almost everywhere, and if $G_{1}, G_{2}, F_{1}, F_{2}$ are replaced with $\widetilde{G}_{1}, \widetilde{G}_{2}, \widetilde{F}_{1}, \widetilde{F}_{2}$, respectively, then equation (7) is satisfied everywhere on $\mathbb{R}_{+}^{2}$.

Pr o of. First, we prove that there exists a unique continuous function $\widetilde{G}_{1}$ which is almost everywhere equal to $G_{1}$ on $\mathbb{R}_{+}$and replacing $G_{1}$ with $\widetilde{G}_{1}$, equation (7) is satisfied almost everywhere. 


\section{KÁROLY LAJKÓ — FRUZSINA MÉSZÁROS}

With the substitution $t=x(\alpha+y)$, we get from (7) the equation

$$
G_{1}(t)=G_{2}\left(y\left(\beta+\frac{t}{\alpha+y}\right)\right)+F_{2}\left(\frac{t}{\alpha+y}\right)-F_{1}(y)
$$

which is satisfied for almost all $(t, y) \in \mathbb{R}_{+}^{2}$. By Fubini's Theorem, it follows that there exists $T^{\prime} \subseteq \mathbb{R}_{+}$of full measure such that for all $t \in T^{\prime}$ equation (8) is satisfied for almost every $y \in D_{t}$, where

$$
D_{t}=\left\{y \in \mathbb{R}_{+} \mid(t, y) \in \mathbb{R}_{+}^{2}\right\}=\mathbb{R}_{+} .
$$

Let us define the functions $g_{1}, g_{2}, g_{3}, h$ in the following way:

$$
\begin{gathered}
g_{1}(t, y)=y\left(\beta+\frac{t}{\alpha+y}\right), \quad g_{2}(t, y)=\frac{t}{\alpha+y}, \quad g_{3}(t, y)=y, \\
h\left(t, y, z_{1}, z_{2}, z_{3}\right)=z_{1}+z_{2}-z_{3},
\end{gathered}
$$

and let us now apply Theorem 1 of J ár a i to (8) with the following casting:

$$
\begin{gathered}
G_{1}=f, \quad G_{2}=f_{1}, \quad F_{2}=f_{2}, \quad F_{1}=f_{3}, \\
Z=Z_{i}=\mathbb{R}, \quad T=Y=X_{i}=\mathbb{R}_{+}, \quad(i=1,2,3) .
\end{gathered}
$$

Hence, the first assumption in Theorem 1 with respect to (8) holds. In the event of fixed $y$, the function $h$ is continuous in other variables, so the second assumption holds, too. Because the functions in equation (8) are measurable, the third assumption is trivially satisfied.

The functions $g_{i}$ are continuous, the partial derivatives

$$
D_{2} g_{1}(t, y)=\frac{t \alpha}{(y+\alpha)^{2}}+\beta, \quad D_{2} g_{2}(t, y)=-\frac{t}{(y+\alpha)^{2}}, \quad D_{2} g_{3}(t, y)=1
$$

are also continuous, so the fourth assumption holds, too.

For each $t \in \mathbb{R}_{+}$there exists a $y \in \mathbb{R}_{+}$such that $(t, y) \in \mathbb{R}_{+}^{2}$ and the partial derivatives do not equal zero in $(t, y)$, so they have rank 1 . Thus, the last assumption is satisfied in Theorem 1.

As a result, from Theorem 1 we get that there exists a unique continuous function $\widetilde{G}_{1}$ which is almost everywhere equal to $G_{1}$ on $\mathbb{R}_{+}$and $\widetilde{G}_{1}, G_{2}, F_{1}, F_{2}$ satisfy equation (7) almost everywhere, which is equivalent to the equation $\widetilde{G}_{1}(x(\alpha+y))+F_{1}(y)=G_{2}(y(\beta+x))+F_{2}(x) \quad$ for almost all $(x, y) \in \mathbb{R}_{+}^{2}$.

Thanks to a similar argument, we can prove the same for the function $G_{2}$.

From equation (9) with the substitution $t=y(\beta+x)$, we get the equation

$$
G_{2}(t)=\widetilde{G}_{1}\left(\left(\frac{t}{y}-\beta\right)(\alpha+y)\right)+F_{1}(y)-F_{2}\left(\frac{t}{y}-\beta\right)
$$




\section{FUNCTIONAL EQUATIONS STEMMING FROM PROBABILITY THEORY}

which with a suitable casting, by Fubini's Theorem, and the fact that the assumptions of Theorem 1 are fulfilled again, gives us that there exists a unique continuous function $\widetilde{G}_{2}$ which is almost everywhere equal to $G_{2}$ on $\mathbb{R}_{+}$and $\widetilde{G}_{1}$, $\widetilde{G}_{2}, F_{1}, F_{2}$ satisfy equation (77) almost everywhere, i.e., $\widetilde{G}_{1}(x(\alpha+y))+F_{1}(y)=\widetilde{G}_{2}(y(\beta+x))+F_{2}(x) \quad$ for almost all $(x, y) \in \mathbb{R}_{+}^{2}$.

There exists such $x_{0}$ that, with the substitution $x=x_{0}$, from equation (10) we get that

$$
F_{1}(y)=\widetilde{G}_{2}\left(y\left(\beta+x_{0}\right)\right)+F_{2}\left(x_{0}\right)-\widetilde{G}_{1}\left(x_{0}(\alpha+y)\right)
$$

holds for almost all $y \in \mathbb{R}_{+}$, and there exists such $y_{0}$ that, with the substitution $y=y_{0}$, we get from equation (10) that

$$
F_{2}(x)=\widetilde{G}_{1}\left(x\left(\alpha+y_{0}\right)\right)+F_{1}\left(y_{0}\right)-\widetilde{G}_{2}\left(y_{0}(\beta+x)\right)
$$

holds for almost all $x \in \mathbb{R}_{+}$. As $\widetilde{G}_{1}, \widetilde{G}_{2}: \mathbb{R}_{+} \rightarrow \mathbb{R}$ are continuous, there exist unique continuous functions $\widetilde{F}_{1}, \widetilde{F}_{2}: \mathbb{R}_{+} \rightarrow \mathbb{R}$, defined by the right-hand side of the last two equalities which are almost everywhere equal to $F_{1}$ and $F_{2}$ on $\mathbb{R}_{+}$, respectively, and if we replace $F_{1}$ and $F_{2}$ with $\widetilde{F}_{1}$ and $\widetilde{F}_{2}$, respectively, the functional equation

$$
\widetilde{G}_{1}(x(\alpha+y))+\widetilde{F}_{1}(y)=\widetilde{G}_{2}(y(\beta+x))+\widetilde{F}_{2}(x)
$$

is satisfied almost everywhere on $\mathbb{R}_{+}^{2}$.

Both sides of (13) define continuous functions on $\mathbb{R}_{+}^{2}$ which are equal to each other on a dense subset of $\mathbb{R}_{+}^{2}$, therefore we get that (13) is satisfied everywhere on $\mathbb{R}_{+}^{2}$.

Further, $G_{1}=\widetilde{G}_{1}, G_{2}=\widetilde{G}_{2}, F_{1}=\widetilde{F}_{1}$ and $F_{2}=\widetilde{F}_{2}$ almost everywhere on $\mathbb{R}_{+}$.

Therefore, it suffices to determine the general continuous solutions $\widetilde{G}_{1}, \widetilde{G}_{2}$, $\widetilde{F}_{1}, \widetilde{F}_{2}: \mathbb{R}_{+} \rightarrow \mathbb{R}$ of equation (13) for all $(x, y) \in \mathbb{R}_{+}^{2}$.

Lemma 2. If the continuous functions $\widetilde{G}_{1}, \widetilde{G}_{2}, \widetilde{F}_{1}, \widetilde{F}_{2}: \mathbb{R}_{+} \rightarrow \mathbb{R}$ satisfy the functional equation (13) for all $(x, y) \in \mathbb{R}_{+}^{2}$, then they are differentiable infinitely many times on $\mathbb{R}_{+}$.

P r o o f. Let equation (13) be written in the form (8):

$$
\widetilde{G}_{1}(t)=\widetilde{G}_{2}\left(y\left(\beta+\frac{t}{\alpha+y}\right)\right)+\widetilde{F}_{2}\left(\frac{t}{\alpha+y}\right)-\widetilde{F}_{1}(y)
$$

and let $[a, b] \subset \mathbb{R}_{+}$be arbitrary and choose the interval $[c, d] \subset \mathbb{R}_{+}$arbitrarily, too. Then $[a, b] \times[c, d] \subset \mathbb{R}_{+}^{2}$ holds. 


\section{KÁROLY LAJKÓ — FRUZSINA MÉSZÁROS}

Integrating (14) with respect to $y$ on $[c, d]$ we get

$$
(d-c) \widetilde{G}_{1}(t)=\int_{c}^{d} \widetilde{G}_{2}\left(y\left(\beta+\frac{t}{\alpha+y}\right)\right) d y+\int_{c}^{d} \widetilde{F}_{2}\left(\frac{t}{\alpha+y}\right) d y-\int_{c}^{d} \widetilde{F}_{1}(y) d y .
$$

We use the substitutions

$$
g_{1}(t, y)=y\left(\beta+\frac{t}{\alpha+y}\right)=u, \quad g_{2}(t, y)=\frac{t}{\alpha+y}=u
$$

in the first and second integral, respectively. We will show that these equations can uniquely be solved for $y$ if $t \in[a, b]$.

In the case $\frac{t}{\alpha+y}=u$, this is clear. In the case $y\left(\beta+\frac{t}{\alpha+y}\right)=u$, this uniqueness is ensured, namely, the derivative of the function $y \rightarrow g_{1}(t, y)$ :

$$
D_{2} g_{1}(t, y)=\beta+\frac{t \alpha}{(\alpha+y)^{2}}
$$

is positive on $[a, b] \times[c, d]$, hence our function is strictly increasing. The solutions

$$
\begin{gathered}
y=\frac{u-t-\alpha \beta+\sqrt{(t-u+\alpha \beta)^{2}+4 \alpha \beta u}}{2 \beta} \doteq \gamma_{1}(t, u), \\
y=\frac{t}{u}-\alpha \doteq \gamma_{2}(t, u)
\end{gathered}
$$

are infinitely many times differentiable functions of $t$ and $u$. Performing the substitutions, we have

$$
\widetilde{G}_{1}(t)=\frac{1}{d-c}\left[\int_{\beta c+\frac{t c}{\alpha+c}}^{\beta d+\frac{t d}{\alpha+d}} \widetilde{G}_{2}(u) D_{2} \gamma_{1}(t, u) d u+\int_{\frac{t}{\alpha+c}}^{\frac{t}{\alpha+d}} \widetilde{F}_{2}(u) D_{2} \gamma_{2}(t, u) d u-C\right],
$$

where

$$
C=\int_{c}^{d} \widetilde{F}_{1}(y) d y
$$

The functions $\widetilde{G}_{2}, \widetilde{F}_{2}$ are at least continuous. Hence, by repeated application of the theorem concerning the differentiation of parametric integrals (see e.g. [2]), the right-hand side is differentiable infinitely many times on $[a, b]$. Since $[a, b]$ is an arbitrary subinterval of $\mathbb{R}_{+}$, we have that $\widetilde{G}_{1}$ is differentiable infinitely many times on $\mathbb{R}_{+}$. The differentiability of $\widetilde{G}_{2}$ can be obtained similarly.

With help of (11) and (12), we can deduce that $\widetilde{F}_{1}$ and $\widetilde{F}_{2}$ are also differentiable infinitely many times on $\mathbb{R}_{+}$. 


\section{FUNCTIONAL EQUATIONS STEMMING FROM PROBABILITY THEORY}

Lemma 3. If the functions $\widetilde{G}_{1}, \widetilde{G}_{2}, \widetilde{F}_{1}, \widetilde{F}_{2}: \mathbb{R}_{+} \rightarrow \mathbb{R}$ satisfy the functional equation (13) for all $(x, y) \in \mathbb{R}_{+}^{2}$ and are twice differentiable on $\mathbb{R}_{+}$, then there exist constants $c_{1}, c_{2}, \gamma, \delta_{1}, \delta_{2}, \delta_{3}, \delta_{4} \in \mathbb{R}$, with $\delta_{1}+\delta_{3}=\delta_{2}+\delta_{4}$ such that

$$
\begin{array}{lll}
\widetilde{G}_{1}(t)=c_{1} \ln t+\gamma t+\delta_{1}, & t \in \mathbb{R}_{+}, \\
\widetilde{G}_{2}(t)=c_{2} \ln t+\gamma t+\delta_{2}, & & t \in \mathbb{R}_{+}, \\
\widetilde{F}_{1}(t)=-c_{1} \ln (t+\alpha)+c_{2} \ln t+\gamma \beta t+\delta_{3}, & & t \in \mathbb{R}_{+}, \\
\widetilde{F}_{2}(t)=c_{1} \ln t-c_{2} \ln (t+\beta)+\gamma \alpha t+\delta_{4}, & & t \in \mathbb{R}_{+} .
\end{array}
$$

P r o o f. Differentiating (13) with respect to $x$, then differentiating the resulting equation with respect to $y$, we get

$$
\begin{aligned}
(\alpha+y) x \widetilde{G}_{1}^{\prime \prime}[(\alpha+y) x]+\widetilde{G}_{1}^{\prime}[(\alpha+y) x] & \\
& =(\beta+x) y \widetilde{G}_{2}^{\prime \prime}[(\beta+x) y]+\widetilde{G}_{2}^{\prime}[(\beta+x) y],
\end{aligned}
$$

where $(x, y) \in \mathbb{R}_{+}^{2}$. It is easy to see that this can hold if and only if

$$
t \widetilde{G}_{1}^{\prime \prime}(t)+\widetilde{G}_{1}^{\prime}(t)=\gamma=s \widetilde{G}_{2}^{\prime \prime}(s)+\widetilde{G}_{2}^{\prime}(s), \quad t, s \in \mathbb{R}_{+}
$$

for some constant $\gamma$.

The general solution to the differential equations

and

$$
t \widetilde{G}_{1}^{\prime \prime}(t)+\widetilde{G}_{1}^{\prime}(t)=\gamma, \quad t \in \mathbb{R}_{+},
$$

$$
s \widetilde{G}_{2}^{\prime \prime}(s)+\widetilde{G}_{2}^{\prime}(s)=\gamma, \quad s \in \mathbb{R}_{+},
$$

have the following forms

$$
\begin{array}{ll}
\widetilde{G}_{1}(t)=c_{1} \ln t+\gamma t+\delta_{1}, & t \in \mathbb{R}_{+}, \\
\widetilde{G}_{2}(s)=c_{2} \ln s+\gamma s+\delta_{2}, & s \in \mathbb{R}_{+},
\end{array}
$$

where

$$
c_{1}, c_{2}, \gamma, \delta_{1}, \delta_{2} \in \mathbb{R}
$$

are arbitrary constants, thus we get (15) and (16). An easy calculation gives the remaining for (17) and (18).

It is easy to see that (15), (16), (17) and (18) satisfy (13) if

$$
\delta_{1}+\delta_{3}=\delta_{2}+\delta_{4} .
$$

Thus, as an immediate consequence of Lemma 1, Theorem 2, Lemma 2 and Lemma 3, for the measurable solution of equation (6) we get the following: 


\section{KÁROLY LAJKÓ — FRUZSINA MÉSZÁROS}

TheOREM 3. The measurable functions $h_{1}, h_{2}, f_{X}, f_{Y}: \mathbb{R}_{+} \rightarrow \mathbb{R}_{+}$satisfy functional equation (6) for almost all $(x, y) \in \mathbb{R}_{+}^{2}$ if and only if

$$
\begin{array}{ll}
h_{1}(x)=x^{c_{1}} \exp \left(\gamma x+\delta_{1}\right) & \text { a.e. } x \in \mathbb{R}_{+}, \\
h_{2}(x)=x^{c_{2}} \exp \left(\gamma x+\delta_{2}\right) & \text { a.e. } x \in \mathbb{R}_{+}, \\
f_{Y}(y)=\frac{y^{c_{2}}}{(y+\alpha)^{c_{1}+1}} \exp \left(\gamma \beta y+\delta_{3}\right) & \text { a.e. } y \in \mathbb{R}_{+}, \\
f_{X}(x)=\frac{x^{c_{1}}}{(x+\beta)^{c_{2}+1}} \exp \left(\gamma \alpha x+\delta_{4}\right) & \text { a.e. } x \in \mathbb{R}_{+},
\end{array}
$$

where $c_{1}, c_{2}, \gamma, \delta_{1}, \delta_{2}, \delta_{3}, \delta_{4} \in \mathbb{R}$ are arbitrary constants with $\delta_{1}+\delta_{3}=\delta_{2}+\delta_{4}$.

Remark 1. The previous theorem shows that $h_{1}$ and $h_{2}$ are gamma densities (with parameters $-\gamma, c_{1}+1$ and $-\gamma, c_{2}+1$, respectively). Thus, $(X, Y)$ has gamma conditionals in this case.

Remark 2. In this special case, the joint density function is of the form

$$
f_{(X, Y)}(x, y)=\exp \left(\delta_{1}+\delta_{3}\right) x^{c_{1}} y^{c_{2}} \exp (\gamma(\alpha x+x y+\beta y))
$$

for almost all $(x, y) \in \mathbb{R}_{+}^{2}$, i.e., the class of all solutions to (2) and (3) (in case $\left.c(y)=\frac{1}{\alpha+y}, d(x)=\frac{1}{\beta+x}\right)$ coincides with the MODEL II gamma conditional class (see [1]).

\subsection{The $\alpha=\beta=0$ case}

From (17), the following equation arises

$$
G_{1}(x y)+F_{1}(y)=G_{2}(x y)+F_{2}(x)
$$

and with the notations

$$
H(t)=G_{1}(t)-G_{2}(t), \quad F(t)=F_{2}(t), \quad G(t)=-F_{1}(t)
$$

we get the equation

$$
H(x y)=F(x)+G(y)
$$

for almost all $(x, y) \in \mathbb{R}_{+}^{2}$, where $F, G, H: \mathbb{R}_{+} \rightarrow \mathbb{R}$ are measurable functions.

Similarly as in Theorem 2, with help of Theorem 1, we can prove the following

TheOREM 4. If the measurable functions $F, G, H: \mathbb{R}_{+} \rightarrow \mathbb{R}$ satisfy equation (19) for almost all $(x, y) \in \mathbb{R}_{+}^{2}$, then there exist unique continuous functions $\widetilde{F}, \widetilde{G}, \widetilde{H}: \mathbb{R}_{+} \rightarrow \mathbb{R}$ such that $\widetilde{F}=F, \widetilde{G}=G$ and $\widetilde{H}=H$ almost everywhere, and if $F, G, H$ are replaced with $\widetilde{F}, \widetilde{G}, \widetilde{H}$, respectively, then equation (19) is satisfied everywhere on $\mathbb{R}_{+}^{2}$. 


\section{FUNCTIONAL EQUATIONS STEMMING FROM PROBABILITY THEORY}

Therefore, we only need the general continuous solutions $\widetilde{F}, \widetilde{G}, \widetilde{H}: \mathbb{R}_{+} \rightarrow \mathbb{R}$ to the Pexider equation

$$
\widetilde{H}(x y)=\widetilde{F}(x)+\widetilde{G}(y)
$$

for all $(x, y) \in \mathbb{R}_{+}^{2}$, which are the following:

$$
\begin{array}{llrl}
\widetilde{H}(t) & =c \ln t+c_{1}+c_{2} & & \left(t \in \mathbb{R}_{+}\right), \\
\widetilde{F}(t) & =c \ln t+c_{1} & & \left(t \in \mathbb{R}_{+}\right), \\
\widetilde{G}(t) & =c \ln t+c_{2} & & \left(t \in \mathbb{R}_{+}\right),
\end{array}
$$

where $c, c_{1}, c_{2} \in \mathbb{R}$ are arbitrary constants. With help of these solutions, Lemma 1 and Theorem 4, we can state the following

TheOREM 5. The measurable functions $h_{1}, h_{2}, f_{X}, f_{Y}: \mathbb{R}_{+} \rightarrow \mathbb{R}_{+}$satisfy functional equation (6) in case $\alpha=\beta=0$ for almost all $(x, y) \in \mathbb{R}_{+}^{2}$ if and only if

$$
\begin{array}{lll}
h_{1}(x)=e^{c_{1}+c_{2}} \exp \left(G_{2}(x)\right) x^{c} & \text { a.e. } & x \in \mathbb{R}_{+}, \\
h_{2}(x)=\exp \left(G_{2}(x)\right) & \text { a.e. } x \in \mathbb{R}_{+}, \\
f_{X}(x)=e^{c_{1}} x^{c-1} & \text { a.e. } x \in \mathbb{R}_{+}, \\
f_{Y}(x)=e^{-c_{2}} x^{-c-1} & \text { a.e. } x \in \mathbb{R}_{+},
\end{array}
$$

where $G_{2}: \mathbb{R}_{+} \rightarrow \mathbb{R}$ is an arbitrary measurable function and $c, c_{1}, c_{2} \in \mathbb{R}$ are arbitrary constants.

Remark 3. The joint density function in this case has the form

$$
f_{(X, Y)}(x, y)=x^{c} e^{G_{2}(x y)+c_{1}} \quad \text { for almost all } \quad(x, y) \in \mathbb{R}_{+}^{2} .
$$

\section{Second problem}

The second inquired case of the general equation (4) is the following. Let the functions $c, d$ be linear, i.e.,

$$
c(y)=\lambda_{1}(\alpha+y), \quad d(x)=\lambda_{2}(\beta+x) \quad(x, y>0),
$$

where $\lambda_{1}, \lambda_{2}$ are positive, $\alpha$ and $\beta$ are non-negative constants.

Hence, from (4), we get the equation

$$
h_{1}\left(\frac{x}{\lambda_{1}(\alpha+y)}\right) \frac{1}{\lambda_{1}(\alpha+y)} f_{Y}(y)=h_{2}\left(\frac{y}{\lambda_{2}(\beta+x)}\right) \frac{1}{\lambda_{2}(\beta+x)} f_{X}(x)
$$

for almost all $(x, y) \in \mathbb{R}_{+}^{2}$, where $h_{1}, h_{2}, f_{X}, f_{Y}: \mathbb{R}_{+} \rightarrow \mathbb{R}_{+}$are measurable unknown functions, $\lambda_{1}, \lambda_{2} \in \mathbb{R}_{+}, \alpha, \beta \geq 0$ are arbitrary constants.

Easy calculation shows the validity of the following technical lemma. 


\section{KÁROLY LAJKÓ — FRUZSINA MÉSZÁROS}

LEMmA 4. The positive measurable functions $h_{1}, h_{2}, f_{X}, f_{Y}$ satisfy equation (21) for almost all $(x, y) \in \mathbb{R}_{+}^{2}$ if and only if the measurable functions $G_{1}, G_{2}, F_{1}$, $F_{2}: \mathbb{R}_{+} \rightarrow \mathbb{R}$ defined by

$$
\begin{array}{rlrl}
G_{1}(t) & =-\ln \left[h_{2}\left(\frac{1}{\lambda_{2} t}\right)\right], & G_{2}(t)=-\ln \left[h_{1}\left(\frac{1}{\lambda_{1} t}\right)\right], & \\
F_{1}(t)=\ln \left[\frac{f_{Y}(t)}{\lambda_{1}(\alpha+t)}\right], & F_{2}(t)=\ln \left[\frac{f_{X}(t)}{\lambda_{2}(\beta+t)}\right], \quad\left(t \in \mathbb{R}_{+}\right)
\end{array}
$$

satisfy the functional equation

$$
G_{1}\left(\frac{x+\beta}{y}\right)+F_{1}(y)=G_{2}\left(\frac{y+\alpha}{x}\right)+F_{2}(x), \quad \text { for almost all } \quad(x, y) \in \mathbb{R}_{+}^{2},
$$

where $\alpha, \beta \geq 0$ are arbitrary constants.

To get the measurable solution of equation (22) (and so (21)) satisfied almost everywhere, we distinguish 2 cases:

(1) $\alpha^{2}+\beta^{2} \neq 0$;

(2) $\alpha=\beta=0$.

\subsection{The $\alpha^{2}+\beta^{2} \neq 0$ case}

In this case, similarly as in Theorem 2, with help of Theorem 1, we can prove the following

TheORem 6. If the measurable functions $G_{1}, G_{2}, F_{1}, F_{2}: \mathbb{R}_{+} \rightarrow \mathbb{R}$ satisfy equation (22) for almost all $(x, y) \in \mathbb{R}_{+}^{2}$, then there exist unique continuous functions $\widetilde{G}_{1}, \widetilde{G}_{2}, \widetilde{F}_{1}, \widetilde{F}_{2}: \mathbb{R}_{+} \rightarrow \mathbb{R}$ such that $\widetilde{G}_{1}=G_{1}, \widetilde{G}_{2}=G_{2}, \widetilde{F}_{1}=F_{1}$ and $\widetilde{F}_{2}=F_{2}$ almost everywhere, and if $G_{1}, G_{2}, F_{1}, F_{2}$ are replaced with $\widetilde{G}_{1}, \widetilde{G}_{2}, \widetilde{F}_{1}, \widetilde{F}_{2}$, respectively, then equation (22) is satisfied everywhere on $\mathbb{R}_{+}^{2}$.

Hence, it is enough to determine the general continuous solutions $\widetilde{G}_{1}, \widetilde{G}_{2}$, $\widetilde{F}_{1}, \widetilde{F}_{2}: \mathbb{R}_{+} \rightarrow \mathbb{R}$ to equation

$$
\widetilde{G}_{1}\left(\frac{x+\beta}{y}\right)+\widetilde{F}_{1}(y)=\widetilde{G}_{2}\left(\frac{y+\alpha}{x}\right)+\widetilde{F}_{2}(x) \quad \text { for all } \quad(x, y) \in \mathbb{R}_{+}^{2} .
$$

The proof of the following lemma goes similarly as that of Lemma 2 ,

Lemma 5. If the continuous functions $\widetilde{G}_{1}, \widetilde{G}_{2}, \widetilde{F}_{1}, \widetilde{F}_{2}: \mathbb{R}_{+} \rightarrow \mathbb{R}$ satisfy the functional equation (23) for all $(x, y) \in \mathbb{R}_{+}^{2}$, then they are differentiable infinitely many times on $\mathbb{R}_{+}$.

From this we get immediately the following 
Lemma 6. If the functions $\widetilde{G}_{1}, \widetilde{G}_{2}, \widetilde{F}_{1}, \widetilde{F}_{2}: \mathbb{R}_{+} \rightarrow \mathbb{R}$ satisfy the functional equation (23) for all $(x, y) \in \mathbb{R}_{+}^{2}$ and are twice differentiable on $\mathbb{R}_{+}$, then $\widetilde{G}_{1}$ and $\widetilde{G}_{2}$ satisfy the differential equations

and

$$
t \widetilde{G}_{1}^{\prime \prime}(t)+\widetilde{G}_{1}^{\prime}(t)=\frac{\gamma}{(\alpha t+\beta)^{2}}, \quad t \in \mathbb{R}_{+}
$$

$$
s \widetilde{G}_{2}^{\prime \prime}(s)+\widetilde{G}_{2}^{\prime}(s)=\frac{\gamma}{(\beta s+\alpha)^{2}}, \quad s \in \mathbb{R}_{+}
$$

with some constant $\gamma$.

P r o o f. Differentiating (23) with respect to $x$, then differentiating the resulting equation with respect to $y$, we get

$$
\begin{aligned}
& x^{2}\left[\frac{x+\beta}{y} \widetilde{G}_{1}^{\prime \prime}\left(\frac{x+\beta}{y}\right)+\widetilde{G}_{1}^{\prime}\left(\frac{x+\beta}{y}\right)\right] \\
& =y^{2}\left[\frac{y+\alpha}{x} \widetilde{G}_{2}^{\prime \prime}\left(\frac{y+\alpha}{x}\right)+\widetilde{G}_{2}^{\prime}\left(\frac{y+\alpha}{x}\right)\right], \quad(x, y) \in \mathbb{R}_{+}^{2} .
\end{aligned}
$$

It is easy to see that this can hold if and only if

$$
(\alpha t+\beta)^{2}\left[t \widetilde{G}_{1}^{\prime \prime}(t)+\widetilde{G}_{1}^{\prime}(t)\right]=\gamma=(\beta s+\alpha)^{2}\left[s \widetilde{G}_{2}^{\prime \prime}(s)+\widetilde{G}_{2}^{\prime}(s)\right], \quad t, s \in \mathbb{R}_{+},
$$

for some constant $\gamma$, which implies the statement of our lemma.

3.1.1. The case $\alpha>0, \beta>0$

Lemma 7. If the functions $\widetilde{G}_{1}, \widetilde{G}_{2}, \widetilde{F}_{1}, \widetilde{F}_{2}: \mathbb{R}_{+} \rightarrow \mathbb{R}$ satisfy the functional equation (23) for all $(x, y) \in \mathbb{R}_{+}^{2}$ and are twice differentiable on $\mathbb{R}_{+}$, then there exist constants $c_{1}, c_{2}, \gamma, d_{1}, d_{2}, d_{3}, d_{4} \in \mathbb{R}$, with $d_{1}+d_{3}=d_{2}+d_{4}$ such that

$$
\begin{array}{lll}
\widetilde{G}_{1}(t)=\left(c_{1}-\frac{\gamma}{\alpha \beta}\right) \ln t+\frac{\gamma}{\alpha \beta} \ln (\alpha t+\beta)+d_{1}, & t \in \mathbb{R}_{+}, \\
\widetilde{G}_{2}(t)=\left(c_{2}-\frac{\gamma}{\alpha \beta}\right) \ln t+\frac{\gamma}{\alpha \beta} \ln (\beta t+\alpha)+d_{2}, & t \in \mathbb{R}_{+}, \\
\widetilde{F}_{1}(t)=c_{1} \ln t+\left(c_{2}-\frac{\gamma}{\alpha \beta}\right) \ln (t+\alpha)+d_{3}, & t \in \mathbb{R}_{+}, \\
\widetilde{F}_{2}(t)=\left(c_{1}-\frac{\gamma}{\alpha \beta}\right) \ln (t+\beta)+c_{2} \ln t+d_{4}, & t \in \mathbb{R}_{+} .
\end{array}
$$




\section{KÁROLY LAJKÓ — FRUZSINA MÉSZÁROS}

P r o of. Because of Lemma 6, $\widetilde{G}_{1}$ and $\widetilde{G}_{2}$ satisfy the differential equations (24) and (25) with some constant $\gamma$, thus we have the following forms

$$
\begin{array}{ll}
\widetilde{G}_{1}(t)=\left(c_{1}-\frac{\gamma}{\alpha \beta}\right) \ln t+\frac{\gamma}{\alpha \beta} \ln (\alpha t+\beta)+d_{1}, \quad t \in \mathbb{R}_{+} \\
\widetilde{G}_{2}(t)=\left(c_{2}-\frac{\gamma}{\alpha \beta}\right) \ln t+\frac{\gamma}{\alpha \beta} \ln (\beta t+\alpha)+d_{2}, \quad t \in \mathbb{R}_{+}
\end{array}
$$

where $c_{1}, c_{2}, d_{1}, d_{2} \in \mathbb{R}$ are arbitrary constants, so $\widetilde{G}_{1}(t)$ and $\widetilde{G}_{2}(t)$ have the required form. An easy calculation shows that the remaining assertions of the lemma are valid as well.

As a consequence of Lemma 4 and Lemma 7 , we get for the measurable solution to equation (21) the following:

TheOREM 7. The measurable functions $h_{1}, h_{2}, f_{X}, f_{Y}: \mathbb{R}_{+} \rightarrow \mathbb{R}_{+}$satisfy functional equation (21) for almost all $(x, y) \in \mathbb{R}_{+}^{2}$ if and only if

$$
\begin{aligned}
& h_{1}(t)=e^{-d_{2}}\left(\lambda_{1} t\right)^{c_{2}}\left(\beta+\lambda_{1} \alpha t\right)^{-\frac{\gamma}{\alpha \beta}} \quad \text { a.e. } \quad t \in \mathbb{R}_{+} \text {, } \\
& h_{2}(t)=e^{-d_{1}}\left(\lambda_{2} t\right)^{c_{1}}\left(\alpha+\lambda_{2} \beta t\right)^{-\frac{\gamma}{\alpha \beta}} \quad \text { a.e. } \quad t \in \mathbb{R}_{+} \text {, } \\
& f_{X}(t)=e^{d_{4}} \lambda_{2} t^{c_{2}}(t+\beta)^{c_{1}-\frac{\gamma}{\alpha \beta}+1} \quad \text { a.e. } \quad t \in \mathbb{R}_{+} \text {, } \\
& f_{Y}(t)=e^{d_{3}} \lambda_{1} t^{c_{1}}(t+\alpha)^{c_{2}-\frac{\gamma}{\alpha \beta}+1} \quad \text { a.e. } \quad t \in \mathbb{R}_{+} \text {, }
\end{aligned}
$$

where $c_{1}, c_{2}, \gamma, d_{1}, d_{2}, d_{3}, d_{4} \in \mathbb{R}$ are arbitrary constants with $d_{1}+d_{3}=d_{2}+d_{4}$.

Remark 4. Theorem $\left[7\right.$ shows that $h_{1}$ and $h_{2}$ are Pearson type VI distributions (with parameters $c_{2}+1, \frac{\gamma}{\alpha \beta}-c_{2}-1$ and $c_{1}+1, \frac{\gamma}{\alpha \beta}-c_{1}-1$, respectively), which are also called beta distributions of the second kind (see [1]). In this case, the marginals $f_{X}$ and $f_{Y}$ have also the same Pearson type VI distribution.

Remark 5. The joint density function is of the form

$$
f_{(X, Y)}(x, y)=\exp \left(d_{3}-d_{2}\right) x^{c_{2}} y^{c_{1}}(\alpha x+\beta y+\alpha \beta)^{-\frac{\gamma}{\alpha \beta}}
$$

for almost all $(x, y) \in \mathbb{R}_{+}^{2}$, thus the class of all solutions to (2) and (3) coincides with an extension of the bivariate Pareto distribution introduced by $\mathrm{M}$ a r d i a (see [1], 6]).

3.1.2. The case $\alpha=0, \beta>0$

Lemma 8. If the functions $\widetilde{G}_{1}, \widetilde{G}_{2}, \widetilde{F}_{1}, \widetilde{F}_{2}: \mathbb{R}_{+} \rightarrow \mathbb{R}$ satisfy the functional equation (23) for all $(x, y) \in \mathbb{R}_{+}^{2}$ and are twice differentiable on $\mathbb{R}_{+}$, then there 
exist constants $c_{1}, c_{2}, \gamma, d_{1}, d_{2}, d_{3}, d_{4} \in \mathbb{R}$, with $d_{1}+d_{3}=d_{2}+d_{4}$ such that

$$
\begin{array}{ll}
\widetilde{G}_{1}(t)=c_{1} \ln t+\frac{\gamma}{\beta^{2}} t+d_{1}, & t \in \mathbb{R}_{+}, \\
\widetilde{G}_{2}(t)=c_{2} \ln t+\frac{\gamma}{\beta^{2} t}+d_{2}, & t \in \mathbb{R}_{+}, \\
\widetilde{F}_{1}(t)=\left(c_{1}+c_{2}\right) \ln t-\frac{\gamma}{\beta t}+d_{3}, & t \in \mathbb{R}_{+}, \\
\widetilde{F}_{2}(t)=c_{1} \ln (t+\beta)+c_{2} \ln t+d_{4}, & t \in \mathbb{R}_{+} .
\end{array}
$$

P r o of. In this case, from Lemma [6 we get that the solutons to (23) can be reduced to the solution to the differential equations

$$
\begin{array}{ll}
t \widetilde{G}_{1}^{\prime \prime}(t)+\widetilde{G}_{1}^{\prime}(t)=\frac{\gamma}{\beta^{2}}, & t \in \mathbb{R}_{+} \\
s \widetilde{G}_{2}^{\prime \prime}(s)+\widetilde{G}_{2}^{\prime}(s)=\frac{\gamma}{(\beta s)^{2}}, & s \in \mathbb{R}_{+}
\end{array}
$$

and hence $\widetilde{G}_{1}$ and $\widetilde{G}_{2}$ have the form

$$
\begin{aligned}
& \widetilde{G}_{1}(t)=c_{1} \ln t+\frac{\gamma}{\beta^{2}} t+d_{1}, \quad t \in \mathbb{R}_{+}, \\
& \widetilde{G}_{2}(t)=c_{2} \ln t+\frac{\gamma}{\beta^{2} t}+d_{2}, \quad t \in \mathbb{R}_{+},
\end{aligned}
$$

where $c_{1}, c_{2}, d_{1}, d_{2} \in \mathbb{R}$ are arbitrary constants, so $\widetilde{G}_{1}(t)$ and $\widetilde{G}_{2}(t)$ have the required form. It can be easily proved that the remaining assertions of the lemma are valid as well.

Thus, for the measurable solution of equation (21) we get the following:

TheOREM 8. The measurable functions $h_{1}, h_{2}, f_{X}, f_{Y}: \mathbb{R}_{+} \rightarrow \mathbb{R}_{+}$satisfy functional equation (21) for almost all $(x, y) \in \mathbb{R}_{+}^{2}$ if and only if

$$
\begin{array}{llll}
h_{1}(t)=e^{-d_{2}}\left(\lambda_{1} t\right)^{c_{2}} e^{-\frac{\lambda_{1} \gamma}{\beta^{2}} t} & \text { a.e. } & t \in \mathbb{R}_{+}, \\
h_{2}(t)=e^{-d_{1}}\left(\lambda_{2} t\right)^{c_{1}} e^{-\frac{\gamma}{\beta^{2} \lambda_{2} t}} & \text { a.e. } & t \in \mathbb{R}_{+}, \\
f_{X}(t)=e^{d_{4}} \lambda_{2}(t+\beta)^{c_{1}+1} t^{c_{2}} & \text { a.e. } & t \in \mathbb{R}_{+}, \\
f_{Y}(t)=e^{d_{3}} \lambda_{1} t^{c_{1}+c_{2}+1} e^{-\frac{\gamma}{\beta t}} & \text { a.e. } & t \in \mathbb{R}_{+},
\end{array}
$$

where $c_{1}, c_{2}, \gamma, d_{1}, d_{2}, d_{3}, d_{4} \in \mathbb{R}$ are arbitrary constants with $d_{1}+d_{3}=d_{2}+d_{4}$.

Remark 6. One can easily get that in this case the joint density function is of the form

$$
f_{(X, Y)}(x, y)=\exp \left(d_{3}-d_{2}\right) x^{c_{2}} y^{c_{1}} e^{-\frac{\gamma}{\beta^{2}} \frac{\beta+x}{y}} \quad \text { for almost all } \quad(x, y) \in \mathbb{R}_{+}^{2} .
$$




\section{KÁROLY LAJKÓ — FRUZSINA MÉSZÁROS}

3.1.3. The case $\alpha>0, \beta=0$

Lemma 9. If the functions $\widetilde{G}_{1}, \widetilde{G}_{2}, \widetilde{F}_{1}, \widetilde{F}_{2}: \mathbb{R}_{+} \rightarrow \mathbb{R}$ satisfy the functional equation (23) for all $(x, y) \in \mathbb{R}_{+}^{2}$ and are twice differentiable on $\mathbb{R}_{+}$, then there exist constants $c_{1}, c_{2}, \gamma, d_{1}, d_{2}, d_{3}, d_{4} \in \mathbb{R}$, with $d_{1}+d_{3}=d_{2}+d_{4}$ such that

$$
\begin{array}{lll}
\widetilde{G}_{1}(t)=c_{1} \ln t+\frac{\gamma}{\alpha^{2} t}+d_{1}, & & t \in \mathbb{R}_{+}, \\
\widetilde{G}_{2}(t)=c_{2} \ln t+\frac{\gamma}{\alpha^{2}} t+d_{2}, & & t \in \mathbb{R}_{+}, \\
\widetilde{F}_{1}(t)=c_{1} \ln t+c_{2} \ln (t+\alpha)+d_{3}, & & t \in \mathbb{R}_{+}, \\
\widetilde{F}_{2}(t)=\left(c_{1}+c_{2}\right) \ln t-\frac{\gamma}{\alpha t}+d_{4}, & & t \in \mathbb{R}_{+} .
\end{array}
$$

P r o of. In this case, the solutions to (23) can be reduced to the solution to the differential equations

$$
\begin{aligned}
t \widetilde{G}_{1}^{\prime \prime}(t)+\widetilde{G}_{1}^{\prime}(t)=\frac{\gamma}{(\alpha t)^{2}}, & t \in \mathbb{R}_{+}, \\
s \widetilde{G}_{2}^{\prime \prime}(s)+\widetilde{G}_{2}^{\prime}(s)=\frac{\gamma}{\alpha^{2}}, & s \in \mathbb{R}_{+},
\end{aligned}
$$

and hence, $\widetilde{G}_{1}$ and $\widetilde{G}_{2}$ have the form

$$
\begin{array}{ll}
\widetilde{G}_{1}(t)=c_{1} \ln t+\frac{\gamma}{\alpha^{2} t}+d_{1}, & t \in \mathbb{R}_{+}, \\
\widetilde{G}_{2}(t)=c_{2} \ln t+\frac{\gamma}{\alpha^{2}} t+d_{2}, & t \in \mathbb{R}_{+},
\end{array}
$$

where $c_{1}, c_{2}, d_{1}, d_{2} \in \mathbb{R}$ are arbitrary constants, so $\widetilde{G}_{1}(t)$ and $\widetilde{G}_{2}(t)$ have the required form. One can easily see that the remaining assertions of the lemma are valid as well.

Thus for the measurable solution to equation (21) we get the following:

TheOREM 9. The measurable functions $h_{1}, h_{2}, f_{X}, f_{Y}: \mathbb{R}_{+} \rightarrow \mathbb{R}_{+}$satisfy functional equation (21) for almost all $(x, y) \in \mathbb{R}_{+}^{2}$ if and only if

$$
\begin{array}{lll}
h_{1}(t)=e^{-d_{2}}\left(\lambda_{1} t\right)^{c_{2}} e^{-\frac{\gamma}{\alpha^{2} \lambda_{1} t}} & \text { a.e. } t \in \mathbb{R}_{+}, \\
h_{2}(t)=e^{-d_{1}}\left(\lambda_{2} t\right)^{c_{1}} e^{-\frac{\gamma \lambda_{2}}{\alpha^{2}} t} & \text { a.e. } t \in \mathbb{R}_{+}, \\
f_{X}(t)=e^{d_{4}} \lambda_{2} t^{c_{1}+c_{2}+1} e^{-\frac{\gamma}{\alpha t}} & \text { a.e. } t \in \mathbb{R}_{+}, \\
f_{Y}(t)=e^{d_{3}} \lambda_{1}(t+\alpha)^{c_{2}+1} t^{c_{1}} & \text { a.e. } t \in \mathbb{R}_{+},
\end{array}
$$

where $c_{1}, c_{2}, \gamma, d_{1}, d_{2}, d_{3}, d_{4} \in \mathbb{R}$ are arbitrary constants with $d_{1}+d_{3}=d_{2}+d_{4}$.

Remark 7. In this case, the joint density function is of the form

$$
f_{(X, Y)}(x, y)=\exp \left(d_{3}-d_{2}\right) x^{c_{2}} y^{c_{1}} e^{-\frac{\gamma}{\alpha^{2}} \frac{\alpha+y}{x}} \quad \text { for almost all } \quad(x, y) \in \mathbb{R}_{+}^{2} .
$$




\subsection{The $\alpha=\beta=0$ case}

From (22) the following equation arises

$$
G_{1}\left(\frac{x}{y}\right)+F_{1}(y)=G_{2}\left(\frac{y}{x}\right)+F_{2}(x)
$$

and with the substitution $x \rightarrow x y$ and the notations

$$
H(t)=F_{2}(t), \quad F(t)=G_{1}(t)-G_{2}\left(\frac{1}{t}\right), \quad G(t)=F_{1}(t)
$$

we get the Pexider equation

$$
H(x y)=F(x)+G(y)
$$

again for almost all $(x, y) \in \mathbb{R}_{+}^{2}$, where $F, G, H: \mathbb{R}_{+} \rightarrow \mathbb{R}$ are measurable functions.

Let us use the result of Theorem 4, hence we only need the general continuous solutions $\widetilde{F}, \widetilde{G}, \widetilde{H}: \mathbb{R}_{+} \rightarrow \mathbb{R}$ to the Pexider equation (20) for all $(x, y) \in \mathbb{R}_{+}^{2}$ (the measureable solutions to the almost everywhere satisfied Pexider equation are almost everywhere equal to these solutions), which are the following:

$$
\begin{aligned}
\widetilde{H}(t) & =c \ln t+c_{1}+c_{2}, & & \left(t \in \mathbb{R}_{+}\right), \\
\widetilde{F}(t) & =c \ln t+c_{1}, & & \left(t \in \mathbb{R}_{+}\right), \\
\widetilde{G}(t) & =c \ln t+c_{2}, & & \left(t \in \mathbb{R}_{+}\right),
\end{aligned}
$$

where $c, c_{1}, c_{2} \in \mathbb{R}$ are arbitrary constants.

With help of these solutions and Lemma 1, we can state the following

TheOREM 10. The measurable functions $h_{1}, h_{2}, f_{X}, f_{Y}: \mathbb{R}_{+} \rightarrow \mathbb{R}_{+}$satisfy functional equation (21) in case $\alpha=\beta=0$ for almost all $(x, y) \in \mathbb{R}_{+}^{2}$ if and only if

$$
\begin{array}{lll}
h_{1}(x)=\exp \left(-G_{2}\left(\frac{1}{\lambda_{1} x}\right)\right) & \text { a.e. } & x \in \mathbb{R}_{+}, \\
h_{2}(x)=e^{c_{1}} \exp \left(-G_{2}\left(\lambda_{2} x\right)\right)\left(\lambda_{2} x\right)^{c} & \text { a.e. } & x \in \mathbb{R}_{+}, \\
f_{X}(x)=e^{c_{1}+c_{2}} \lambda_{2} x^{c+1} & \text { a.e. } & x \in \mathbb{R}_{+}, \\
f_{Y}(x)=e^{c_{2}} \lambda_{1} x^{c+1} & \text { a.e. } & x \in \mathbb{R}_{+},
\end{array}
$$

where $G_{2}: \mathbb{R}_{+} \rightarrow \mathbb{R}$ is an arbitrary measurable function and $c, c_{1}, c_{2} \in \mathbb{R}$ are arbitrary constants.

Remark 8. The joint density function in this case has the form

$$
f_{(X, Y)}(x, y)=y^{c} e^{-G_{2}\left(\frac{y}{x}\right)+c_{2}} \quad \text { for almost all } \quad(x, y) \in \mathbb{R}_{+}^{2} .
$$




\section{KÁROLY LAJKÓ — FRUZSINA MÉSZÁROS}

\section{REFERENCES}

[1] ARNOLD, B. C.-CASTILlO, E.-SARABIA, J. M.: Conditional Specification of Statistical Models. Springer-Verlag, New York, 1999.

[2] DiEUdonnÉ, J.: Grundzüge der Modernen Analysis. Friedr. Vieweg und Sohn, Braunschweig, 1971.

[3] JÁRAI, A.: Measurable solutions of functional equations satisfied almost everywhere, Math. Pannon. 10 (1999), 103-110.

[4] JÁRAI, A.: Regularity Properties of Functional Equations in Several Variables, in: Adv. Math. (Dordr.), Vol. 8, Kluwer Acad. Publ., Dordrecht, 2005.

[5] LAJKÓ, K.: Functional equations in the theory of conditionally specified distributions, Publ. Math. Debrecen 58 (2001), 241-248.

[6] MARDIA, K. V.: Multivariate Pareto distributions, Ann. Math. Stat. 33 (1962), 1008-1015.

[7] NARUMI, S.: On the general form of bivariate frequency distributions which are mathematically possible when regression and variation are subjected to limiting conditions I, II, Biometrika 15 (1923), 77-88, 209-221.

Received November 26, 2008

Institute of Mathematics

University of Debrecen

P. O. Box 12

HU-4010 Debrecen

HUNGARY

E-mail: lajko@math.klte.hu mefru@math.klte.hu 